\title{
IMPORTANCE OF DIFFERENT \\ CHARACTERISTIC OF THE CORTICOSPINAL TRACT BASED ON TRACTOGRAPHY AND CADAVERIC MICRODISSECTION
}

\author{
Ali Karadag1, Muyassar Mirkhasilova², Erik H. Middlebrooks ${ }^{3,4}$, \\ Yagmurlu $^{5}$, Mahmut Camlar ${ }^{1}$, Sami Bardakci ${ }^{1}$, Mehmet Senoglu', \\ ${ }^{1}$ Health Science University, Tepecik Research and Training Hospital, Department of Neurosurgery, Izmir, Turkey. \\ 2 Levent Hospital, Department of Neurosurgery, Istanbul, Turkey. \\ ${ }^{3}$ Department of Radiology, Mayo Clinic, Jacksonville, FL, USA. \\ ${ }^{4}$ Department of Neurosurgery, Mayo Clinic, Jacksonville, FL, USA. \\ ${ }^{5}$ Department of Neurological Surgery, University of Virginia Health System, Charlottesville, Virginia \\ ${ }^{6}$ Department of Neurosurgery, KTO Karatay University, Medicana International Izmir Hospital, Izmir Turkey.
}

Address for Correspondence: Ali Karadag M.D., E-mail: egealikaradag@gmail.com

Received: 29.03.2021; Accepted: 06.04.2021; Available Online Date: 27.05.2021

(C) Copyright 2021 by Dokuz Eylül University, Institute of Health Sciences - Available online at https://dergipark.org.tr/en/pub/jbachs

Cite this article as: Karadag A, Mirkhasilova M, Middlebrooks EH, Yagmurlu K, Camlar M, Bardakci S, Senoglu M. Importance of Different Characteristic of the Corticospinal Tract Based on and Cadaveric Microdissection. J Basic Clin Health Sci 2021; 2: 65-72.

\begin{abstract}
Purpose: Microsurgical anatomy of the corticospinal tract, its functional role and crucial points in differential diagnosis were evaluated. There is no consensus about its differences and cerebral origin of the corticospinal tract. Tractography and cadaver dissection can help to investigate the characteristics of the corticospinal tract. Also, amyotrophic lateral sclerosis is hard to diagnose as it has common symptoms and signs with other disorders.

Methods: Three formalin-fixed human brains (six sides) were dissected by the Klingler technique in a stepwise manner from the lateral to medial and superior to inferior under $6 x-40 x$ magnification using a surgical microscope. All stages of the dissection were photographed using three-dimensional method. Lastly, we present a patient with the sign of drop foot who underwent electromyographical and radiological examination, diagnosed as atypic amyotrophic lateral sclerosis.

Results: The connections of the corticospinal tract, in particular the travel in the in trajectories of stepwise in manner cerebral origin. We demonstrated a case report with anatomic correlation to define the damage of corticospinal tract in variable levels. Crucial landmarks, connections, eloquent brain areas that related to the corticospinal tract were emphasized.
\end{abstract}

Conclusion: So that pointing the importance of such disorders to keep in mind as not to move forward with incorrect operation decision. Trajectory of one of the largest desending pathway, corticospinal tract and the relationship with different diagnosis should be considered.

Keywords: Amyotrophic lateral sclerosis, corticospinal tract, differential diagnosis, foot drop

\section{INTRODUCTION}

Major descending neural pathway in the human brain is corticospinal (CST) or pyramidal tract.(1-3) Beside being the major motor pathway for cortical control of voluntary distal movements especially fine motor movements of hand $(1,4,5)$, the CST fibers with 
different cortical origins have different function, fibers amount and recovery mechanism $(6,7)$. Differences in function and fibers amount of CST due to cortical origin and the course of CST have reported by many previous studies using animal brains (8-12). Lately improvement in magnetic resonance imaging (MRI) examination techniques like diffusion tensor imaging (DTI) have opened new avenues of research and have allowed non-invasively mapping this tract in humans because DTI allows to isolate the CST from adjacent structures (13-20). However, review articles from cadaveric anatomical dissection viewpoint are lacking. The word of Amyotrophic lateral sclerosis (ALS) originates from; " $A$ " as a negative meaning, "myo" as muscle, "trophic" as related to nutrition, due to effected lateral side of the spinal cord and "sclerosis" for the hardness (21). ALS is the mostly seen neuromuscular disorder with the incidence of $1 / 100.000$ and prevalence of $4-5 / 100.000$. This disorder is mostly sporadic which is seen between the 4th and 5th decades of life with the male dominancy. Hereditary factors do not have any effect in the $90 \%$ of patients (22).

In current study, we attempted to define characteristics of CST by correlating anatomical fiber microdissection and DTI of these fibers. Also, we would like to take attention what to do and thinking about neuromuscular disorders in such patients administered with foot drop that is mostly seen in lumbar disc herniation.

\section{MATERIALS AND METHODS}

Three formalin-fixed human brains (6 sides) were dissected using the fiber dissection technique that was first described by Klingler (23). The specimens were frozen for 15 days at $-15{ }^{\circ} \mathrm{C}$ according to Klingler's technique and waited in $70 \%$ alcohol solution between the dissection sessions. The freezing process facilitates the dissection of the fiber tracts. Next, the dissection was performed from lateral to medial, anterior to posterior under $x 6$ to $\times 40$ magnifications provided by a Zeiss Surgical Microscope (Carl Zeiss AG, Germany). After removal of the arachnoid mater and vascular structures, the hemispheres were dissected in a stepwise systematic manner, from lateral to medial. Carefully examination of the anatomy of the CST, the topographic location of the related fiber tracts were investigated in detail. Two and three-dimensional anatomic and surgical pictures were obtained and processed by Canon EOS 550 digital camera (Canon, Tokyo, Japan).

\section{RESULTS}

\section{Anatomic dissection}

The dissection was performed from lateral to medial and anterior to posterior. The sulco-gyral anatomy of each specimen was carefully dissected and special attention was given to the CST and its connections. After removing the cortical grey matter around the origins of the CST, we continued to the deep sulcus area and the subcortex was posed as a thinner layer. The CST is a descending large tract of the central nervous system and plays a critical role in movements. These fibers lie in extension that starts at the level of corona radiata to the internal capsule and cerebral peduncle. Also, they travel in the pons to reach the medulla oblongata $(24,25)$ (Figure $1 \mathrm{~A}$, $B, C)$. Defining the CST in the pons with dissection starting in the midbrain is a challenging (Figure 1D, $\mathrm{E})$, but when dissected from below at the medullary pyramid, it could be followed into the middle third of the cerebral peduncle. In the pons, the coursing of the CST stays anteromedial and CST can be exposed after removal the superficial transpontine fibers (Figure 1D). The part of the corticobulbar tract that travels dorsal to the CST, it connects with the related cranial nerve nuclei. The corticopontine fibers end at the pontine nuclei that are scattered anterior and posterior to the corticospinal and corticobulbar tracts (25). The CST is related to the LMN and UMN in the central nervous system. The main part of CST is formed by Primary motor area, the premotor area, and the supplementary motor area of the frontal lobe. Primary sensory area, the parietal operculum and the parietal cortex are known as the other areas. Projections of CST through the centrum semiovale show predictable characteristic and has a straight coursing through the cortex of the brain (Figure 1A, $B, C)$. The CST then enters the midbrain through the cerebral peduncle, where it occupies the middle third of the crus cerebri (Figure 1D, E). The CST therefore can be defined as a bundle of axons; the main cells from which they stem reside in the cerebral cortex. These neurones are named as UMN when they descend from the cortex, or from the brainstem, to synapse on neurones within the brainstem or spinal cord. This is in contrast to neurones from the brainstem or spinal cord that originates from the anterior horn lamina IX, known as LMN. These neurones connections directly on muscle fibres groups (motor units), and provide extra input from UMN's to other neurones. 

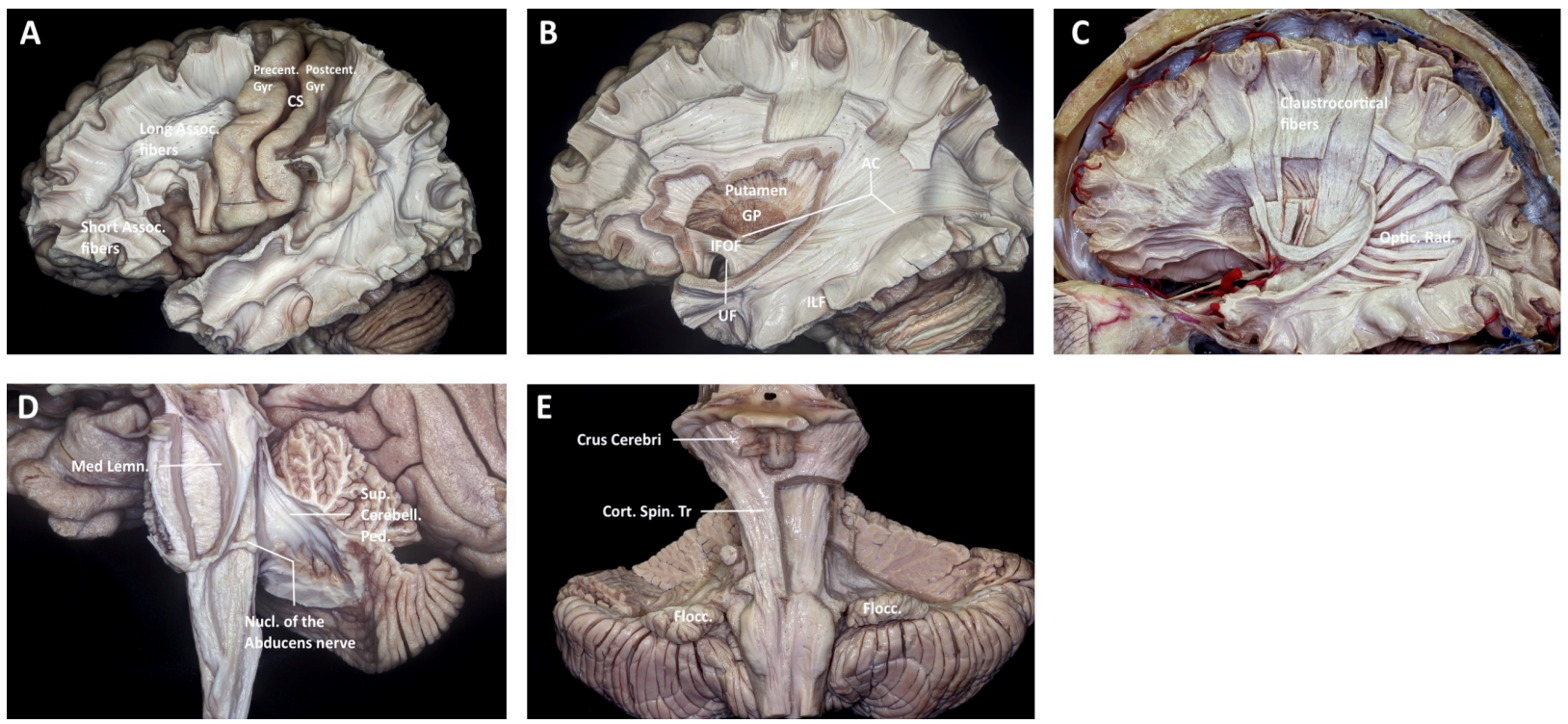

Figure 1. Lateral view of the left hemisphere. Fiber dissection around the primary motor and the central sulcus, subcortical deep white matter fibers and brain stem. The primary motor and sensory regions around the central sulcus. Subcortical deep white matter fibers. The pre-and postcentral gyri form the central lobe. After removal the cerebral cortex including the gray matter and adjacent short association fibers exposed the long association fibers (A). Putamen, GP, ILF, IFOF and UF are exposed. Corona radiata is located just above the putamen and formed by the internal and external capsules (B). The claustrocortical fibers lie between the supplementary motor area and posterior parietal lobe (C). Lateral view of the brainstem. Removal the left superior cerebellar peduncle and dentate nucleus provides an exposure for the medial surface of the superior cerebellar peduncle and dentate nucleus in the right lateral wall of the fourth ventricle. The lateral wall of the superior half of the fourth ventricle is formed by superior cerebellar peduncle (D). Anterior view of the brainstem. The CST is observed at the right and left of the midline after removal the all the superficial transpontine fibers $(E)$.

Abbreviations: AC, anterior commissure; CS, central sulcus; CST, corticospinal tract; GP, globus pallidus; IFOF, inferior frontooccipital fascicle; ILF, inferior longitudinal fascicle; UF, uncinate fascicle

\section{Case description}

Fifty-five year-old female patient administered with 15 days of weakness on the left foot. She was smoking 30 years/1 package and had chronic bronchitis in her anamnesis. Neurological examination was normal in upper extremity. Lower extremity examination showed a muscle strength of right plantar flexion and dorsiflexion as $4 / 5$, left foot plantar and dorsiflexion as $1 / 5$, left knee and hip flexion as $4 / 5$. Laboratory findings were normal. Lumbar MRI showed a degenerated disc on L4-L5 and mild lumbar stenosis at the level of L3-L4 and L5-S1. MRI showed the disc herniation in T2 weighted sagittal image (Figure 2A) and level L1-L2 disc herniation which compressions of the disc to the right structures in T2 weighted axial image (Figure 2B), and level L4-L5 disc herniation which compressions of the disc to the right structures in $\mathrm{T} 2$ weighted axial image (Figure $2 \mathrm{C}$ ).

Neurological examination and radiographic images are compared, we found nothing related to the patient foot drop. We planned electromyography (EMG). EMG supported the ALS diagnosis a with detection chronic neurogenic findings with active denervation discharges related to anterior horn upper neuron damage. We transferred the patient to neurology department for ALS treatment. After electrophysiological examination we focused on the intracranial pathologies and investigated the damage location of the CST according to the symptoms of the patient DTI showed the trajectory of the CST in sagittal and coronal MRI (Figure 2D, E).

\section{DISCUSSION}

CST is the major descending neural pathway in the human brain and studies have suggested that it is unique to mammals (1-4). It is associated with fine motor skills of distal extremities, particularly the activities of hand $(1,4,5)$. Various cortical origins for CSTs are primary motor cortex, secondary motor area and parietal cortex $(7,26,27)$. CSTs with different cortical origins have different function, fiber amount and recovery mechanism $(6,7)$. Differences in function and fibers amount of CST due to cortical origin have been reported by many previous studies 

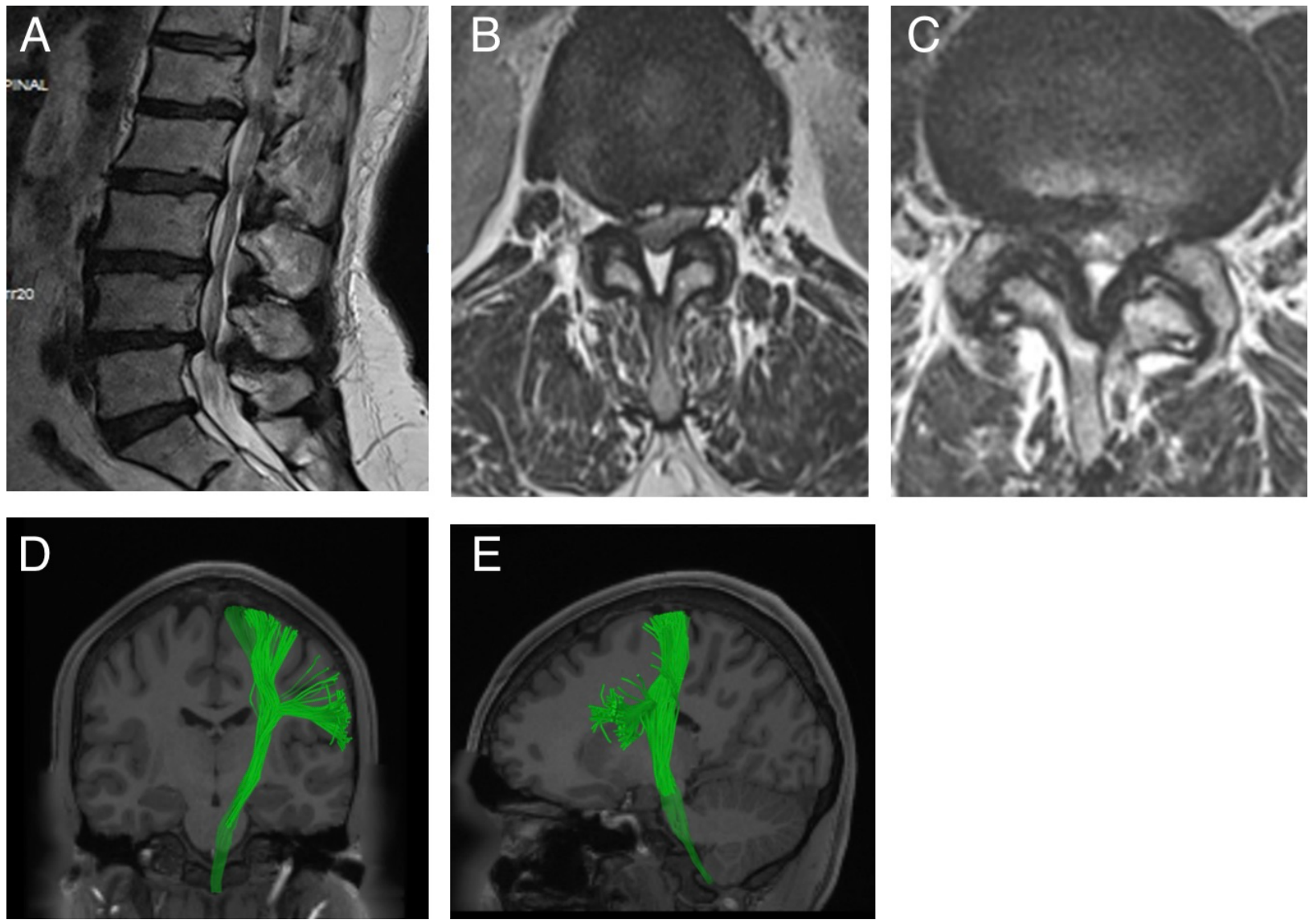

Figure 2. A, L 1-2 and L 4-5 intervertebral disc protrusions in sagittal plane of lumbar magnetic resonance imaging (A). L1-L2 level axial plane MRI (B). L4-L5 level axial plane MRI (C). Trajectory and termination of the CST on DTI (D, E).

Abbreviations: CST, corticospinal tract; DTI, diffusion tensor imaging; L, lumbar; MRI, magnetic resonance imaging.

using animal brains (8-12). On the other hand, DTI allows visualisation of this tract in humans in-vivo (28).

After originating from the cortex, CST then come together as compact bundles to travel through the internal capsule and cerebral peduncles $(29,30)$. Internal capsule is important heterogenous connectivity system. In series of papers, CST has been localised in the posterior part of the posterior limb of the internal capsule $(13,29,31-33)$. Integrity of inferior internal capsule genu is important for fine hand motor function(34). DTI study on 33 healthy subjects suggests CST become more compact and occupying smaller area within pons while passing through pons as it descends (35). While traveling downward the majority of CST axons decussate to the contralateral side forming the pyramidal decussation within medulla; this have critical importance as it means one side of body is controlled by contralateral side of brain $(1,2,5,18,36,37)$.
These crossed lateral fibers occupy approximately $90 \%$ of total CST. The remaining percentage is made up from uncrossed lateral and (uncrossed) ventral fibers $(38,39)$. Existence of uncrossed lateral CST is controversial and size of it varies in every individual (40). In one study researchers was able to find uncrossed lateral CST in only six of sixty human brains (41). While extending caudally; crossed lateral fibers reach till sacral segment but anterior fibers reach only to the upper thoracic cord and rarely descends below the thoracic spinal cord $(1,2,5,18$, $36,37)$. The exact functional roles of these three separate CSTs are unknown but still we can assume they have distinct functional property according to previous studies: lateral CST is mandatory for hand movements and distal muscles are controlled mainly by this tract whereas proximal muscles are controlled by anterior CST and other neural tracts like corticoreticulospinal tract $(1,5,42-46)$. 
Non-CST descending motor pathways, extrapyramidal system includes reticulospinal, vestibulospinal, rubrospinal and tectospinal tracts (5, $38,47,48)$. The cortico-reticulospinal and vestibulospinal tracts are involved in postural control due to axial muscle innervation (48). Tectospinal tract originates from superior colliculus and as a reflex orients head and eyes towards the sound or visual stimuli (49). Vertebrates that use hands or pectoral fins for locomotion have well developed rubrospinal tract. However, in humans it was regressed as a consequence of taking up by CST (50-52).

Clinically, the corticospinal tract is important in ischemic infarcts, rehabilitation, and various neurodegenerative disorders like motor neuron disease which represents wide spectrum expanding group of disorders including ALS $(53,54)$. These progressive diseases characterized by the combined degeneration of lower motor neuron (LMN) and upper motor neuron (UMN); and despite extensive ongoing studies, the diagnosis still remains clinical one. Proving UMN-CST involvement, thus to be able to differentiate motor neuron disease from other illnesses with similar clinic, is very challenging. Lately MRI examination techniques like DTI have allowed non-invasively mapping this tract in humans with the ability to isolate the CST from adjacent structures (13$20,24)$. However, review articles of cadaveric fiber dissection of CST, are lacking.

ALS is a disorder related to UMN and LMN denervation, showing signs and symptoms related to motor neuron and corticospinal neuron involvement. Fatal complications include respiratory depression, bronchopneumonia and asphyxia. Survival of patients, who have ALS type of faster progression, is approximately three to five years (55). Even if there are benign prototypes of ALS, are mostly fatal and differential diagnose is the most important point for the treatment. As it is stated before, it has nonspecific signs and symptoms with asymmetric progression causing it hard to diagnose. EMG and excessive reduction of cerebrospinal fluid proteins (cystatin $\mathrm{C}$, transthyretin and neuroendocrine protein 7B2 carboxyl terminal part) has an important role in diagnose $(56,57)$. When we review the literature for the pathogenesis of ALS, there are multiple factors causing this syndrome. Excitotoxicity, oxidative stress, disturbed neurotropism and cytoskeletal anomalies are the five main topics about causes of ALS in the literature $(55,58,59)$. Excitotoxicity is due to partial glutamate dehydrogenase insufficiency as we seen in the literature. Autoimmunity pathogenesis is related with the $\mathrm{Cu} / \mathrm{Zn}$ superoxide dismutase gene (SOD1) mutation causing oxidative stress ending up with ALS. ALS pathogenesis is also related with heavy metals such as lead, copper, arsenic, aluminum and some viruses (polio and coxsackie virus) $(55,58-62)$. The CST fibers primary motor cortex, the secondary motor area, and the somatosensory cortex, have a special way in the internal capsule and the middle portion of the cerebral peduncle. These fibers bundle occurs the pyramidal tract on the ventral surface of the medulla oblongata after decussate of pontine nuclei between medulla oblongata and spinal cord (63). Therefore, CST fibers are related with arm and leg motor control. Motor deficit of lower extremity may be seen related with motor neuron disease. For this reason, if there is a mismatch between mechanic vertebral reasons and characteristic of motor deficit, the amyotrophic lateral sclerosis should be considered as a possible diagnosis. The cellular loss and damage are usually related only with pyramidal cortex in amyotrophic lateral sclerosis. In addition, MRI and DTI combined imaging can be used for detecting the axonal degeneration of corticospinal tract $(64,65)$. In this case who has foot drop, we first thought of the lumbar disc herniation. Whereas, EMG findings of fibrillation, positive sharp waves with the fluctuating potential findings leads our diagnose as ALS. We stated how hard to diagnose ALS as it has nonspecific symptoms at first stages, just like in our patients atypic drop foot sign due to disorder, and as they are related with more than one pathogenesis.

\section{CONCLUSION}

In this study we attempted to define characteristics of CST by using both anatomical fiber microdissection and DTI of these fibers. Variable eloquent brain areas are the origins of the CST. CST has many connections with neurones synapse on LMNs to effect motor movements. These LMNs are essential for our maintenance and ambulation. We thought that the drop foot is caused from the CST fibers damage with ALS. The most important point is to examine the patient carefully with the right steps to reach the correct diagnose.

Conflict of Interest: The authors declare no competing financial interests and no sources of funding and support, including any for equipment and medications.

Peer-review: Externally peer-reviewed. 


\section{REFERENCES}

1. Davidoff RA. The pyramidal tract. Neurology. 1990;40(2):332-9.

2. Nyberg-Hansen R. ["The pyramidal tract syndrome" in man in the light of experimental investigations]. Tidsskr Nor Laegeforen. 1968;88(1):8-14.

3. Verhaart WJ. The pyramidal tract. Its structure and functions in man and animals. World Neurol. 1962;3:43-53.

4. Heffner RS, Masterton RB. The role of the corticospinal tract in the evolution of human digital dexterity. Brain Behav Evol. 1983;23(34):165-83.

5. York DH. Review of descending motor pathways involved with transcranial stimulation. Neurosurgery. 1987;20(1):70-3.

6. Natali AL, Reddy V, Bordoni B. Neuroanatomy, Corticospinal Cord Tract. StatPearls. Treasure Island (FL)2020.

7. Seo JP, Jang SH. Different characteristics of the corticospinal tract according to the cerebral origin: DTI study. AJNR Am J Neuroradiol. 2013;34(7):1359-63.

8. Galea MP, Darian-Smith I. Multiple corticospinal neuron populations in the macaque monkey are specified by their unique cortical origins, spinal terminations, and connections. Cereb Cortex. 1994;4(2):166-94.

9. Lemon RN, Maier MA, Armand J, Kirkwood PA, Yang HW. Functional differences in corticospinal projections from macaque primary motor cortex and supplementary motor area. Adv Exp Med Biol. 2002;508:425-34.

10. Maier MA, Armand J, Kirkwood PA, Yang HW, Davis JN, Lemon RN. Differences in the corticospinal projection from primary motor cortex and supplementary motor area to macaque upper limb motoneurons: an anatomical and electrophysiological study. Cereb Cortex. 2002;12(3):281-96.

11. Russell JR, Demyer W. The quantitative corticoid origin of pyramidal axons of Macaca rhesus. With some remarks on the slow rate of axolysis. Neurology. 1961;11:96-108.

12. Toyoshima K, Sakai H. Exact cortical extent of the origin of the corticospinal tract (CST) and the quantitative contribution to the CST in different cytoarchitectonic areas. A study with horseradish peroxidase in the monkey. J Hirnforsch. 1982;23(3):257-69.
13. Chenot $Q$, Tzourio-Mazoyer N, Rheault $F$, Descoteaux M, Crivello F, Zago $L$, et al. A population-based atlas of the human pyramidal tract in 410 healthy participants. Brain Struct Funct. 2019;224(2):599-612.

14. Frey D, Strack V, Wiener E, Jussen D, Vajkoczy $P$, Picht T. A new approach for corticospinal tract reconstruction based on navigated transcranial stimulation and standardized fractional anisotropy values. Neuroimage. 2012;62(3):1600-9.

15. Holodny Al, Gor DM, Watts R, Gutin PH, Ulug AM. Diffusion-tensor MR tractography of somatotopic organization of corticospinal tracts in the internal capsule: initial anatomic results in contradistinction to prior reports. Radiology. 2005;234(3):649-53.

16. Kunimatsu A, Aoki $S$, Masutani $Y$, Abe $O$, Hayashi $\mathrm{N}$, Mori $\mathrm{H}$, et al. The optimal trackability threshold of fractional anisotropy for diffusion tensor tractography of the corticospinal tract. Magn Reson Med Sci. 2004;3(1):11-7.

17. Lee DH, Lee DW, Han BS. Topographic organization of motor fibre tracts in the human brain: findings in multiple locations using magnetic resonance diffusion tensor tractography. Eur Radiol. 2016;26(6):1751-9.

18. O. AM. An Essay on the Human Corticospinal Tract: History, Development, Anatomy, and Connections. Neuroanatomy. 2011;10:1-4.

19. Reich DS, Smith SA, Jones CK, Zackowski KM, van Zijl PC, Calabresi PA, et al. Quantitative characterization of the corticospinal tract at $3 \mathrm{~T}$. AJNR Am J Neuroradiol. 2006;27(10):2168-78.

20. Verstynen T, Jarbo K, Pathak S, Schneider W. In vivo mapping of microstructural somatotopies in the human corticospinal pathways. J Neurophysiol. 2011;105(1):336-46.

21. Otomo A, Pan L, Hadano S. Dysregulation of the autophagy-endolysosomal system in amyotrophic lateral sclerosis and related motor neuron diseases. Neurol Res Int. 2012;2012:498428.

22. Durand J, Amendola J, Bories C, Lamotte d'Incamps B. Early abnormalities in transgenic mouse models of amyotrophic lateral sclerosis. $J$ Physiol Paris. 2006;99(2-3):211-20.

23. J. K. Erleichterung der makroskopischen Praeparation des Gehirns durch den Gefrierprozess. Schweiz Arch Neurol Psychiatr 1935;36:247-56. 
24. Javed K, Reddy V, Lui F. Neuroanatomy, Lateral Corticospinal Tract. StatPearls. Treasure Island (FL)2021.

25. Yagmurlu K, Rhoton AL, Jr., Tanriover N, Bennett JA. Three-dimensional microsurgical anatomy and the safe entry zones of the brainstem. Neurosurgery. 2014;10 Suppl 4:602-19; discussion 19-20.

26. Bozkurt B, Yagmurlu K, Middlebrooks EH, Karadag A, Ovalioglu TC, Jagadeesan B, et al. Microsurgical and Tractographic Anatomy of the Supplementary Motor Area Complex in Humans. World Neurosurg. 2016;95:99-107.

27. Kumar A, Juhasz C, Asano E, Sundaram SK, Makki MI, Chugani DC, et al. Diffusion tensor imaging study of the cortical origin and course of the corticospinal tract in healthy children. AJNR Am J Neuroradiol. 2009;30(10):1963-70.

28. Biceroglu H, Karadag A. Neuroanatomical Aspects of the Temporo-Parieto-Occipital Junction and New Surgical Strategy to Preserve the Associated Tracts in Junctional Lesion Surgery: Fiber Separation Technique. Turk Neurosurg. 2019;29(6):864-74.

29. Kretschmann HJ. Localisation of the corticospinal fibres in the internal capsule in man. J Anat. 1988;160:219-25.

30. Welniarz Q, Dusart I, Roze E. The corticospinal tract: Evolution, development, and human disorders. Dev Neurobiol. 2017;77(7):810-29.

31. Ebeling U, Reulen HJ. Subcortical topography and proportions of the pyramidal tract. Acta Neurochir (Wien). 1992;118(3-4):164-71.

32. Englander RN, Netsky MG, Adelman LS. Location of human pyramidal tract in the internal capsule: anatomic evidence. Neurology. 1975;25(9):823-6.

33. Ross ED. Localization of the pyramidal tract in the internal capsule by whole brain dissection. Neurology. 1980;30(1):59-64.

34. Sullivan EV, Zahr NM, Rohlfing T, Pfefferbaum A. Fiber tracking functionally distinct components of the internal capsule. Neuropsychologia. 2010;48(14):4155-63.

35. Seo JP, Jang SH. Characteristics of corticospinal tract area according to pontine level. Yonsei Med J. 2013;54(3):785-7.

36. Jang $\mathrm{SH}$. The role of the corticospinal tract in motor recovery in patients with a stroke: a review. NeuroRehabilitation. 2009;24(3):285-90.
37. Nathan PW, Smith MC, Deacon P. The corticospinal tracts in man. Course and location of fibres at different segmental levels. Brain. 1990;113 ( Pt 2):303-24.

38. Jang $\mathrm{SH}$. The corticospinal tract from the viewpoint of brain rehabilitation. J Rehabil Med. 2014;46(3):193-9.

39. Karadag A, Senoglu M, Middlebrooks EH, Kinali $B$, Guvencer $M$, Icke $C$, et al. Endoscopic endonasal transclival approach to the ventral brainstem: Radiologic, anatomic feasibility and nuances, surgical limitations and future directions. J Clin Neurosci. 2020;73:264-79.

40. Fulton JF, Sheehan D. The Uncrossed Lateral Pyramidal Tract in Higher Primates. J Anat. 1935;69(Pt 2):181-7.

41. Nyberg-Hansen R, Rinvik E. Some comments on the pyramidal tract, with special reference to its individual variations in man. Acta Neurol Scand. 1963;39:1-30.

42. Cho SH, Kim SH, Choi BY, Cho SH, Kang JH, Lee $\mathrm{CH}$, et al. Motor outcome according to diffusion tensor tractography findings in the early stage of intracerebral hemorrhage. Neurosci Lett. 2007;421(2):142-6.

43. Matsuyama K, Mori F, Nakajima K, Drew T, Aoki $\mathrm{M}$, Mori S. Locomotor role of the corticoreticularreticulospinal-spinal interneuronal system. Prog Brain Res. 2004;143:239-49.

44. Nudo RJ, Masterton RB. Descending pathways to the spinal cord, III: Sites of origin of the corticospinal tract. J Comp Neurol. 1990;296(4):559-83.

45. Schaechter JD, Fricker ZP, Perdue KL, Helmer $K G$, Vangel MG, Greve DN, et al. Microstructural status of ipsilesional and contralesional corticospinal tract correlates with motor skill in chronic stroke patients. Hum Brain Mapp. 2009;30(11):3461-74.

46. Smania N, Paolucci S, Tinazzi M, Borghero A, Manganotti $P$, Fiaschi $A$, et al. Active finger extension: a simple movement predicting recovery of arm function in patients with acute stroke. Stroke. 2007;38(3):1088-90.

47. Jang SH, Lee SJ. Corticoreticular Tract in the Human Brain: A Mini Review. Front Neurol. 2019;10:1188.

48. Nudo RJ, Masterton RB. Descending pathways to the spinal cord: II. Quantitative study of the tectospinal tract in 23 mammals. J Comp Neurol. 1989;286(1):96-119. 
49. Reynolds N, Al Khalili Y. Neuroanatomy, Tectospinal Tract. StatPearls. Treasure Island (FL)2020.

50. G. S, C. W. Spinal Cord: Connections. The Human Nervous System(3rd edition). 2012; Chapter 7 233-58.

51. Onodera S, Hicks TP. A comparative neuroanatomical study of the red nucleus of the cat, macaque and human. PLoS One. 2009;4(8):e6623.

52. ten Donkelaar HJ. Evolution of the red nucleus and rubrospinal tract. Behav Brain Res. 1988;28(1-2):9-20.

53. Rajagopalan V, Pioro EP. Differential involvement of corticospinal tract (CST) fibers in UMN-predominant ALS patients with or without CST hyperintensity: A diffusion tensor tractography study. Neuroimage Clin. 2017;14:574-9.

54. Yin H, Cheng SH, Zhang J, Ma L, Gao Y, Li D, et al. Corticospinal tract degeneration in amyotrophic lateral sclerosis: a diffusion tensor imaging and fibre tractography study. Ann Acad Med Singapore. 2008;37(5):411-5.

55. Eisen A. Amyotrophic lateral sclerosis is a multifactorial disease. Muscle Nerve. 1995;18(7):741-52.

56. Leigh PN, Ray-Chaudhuri K. Motor neuron disease. J Neurol Neurosurg Psychiatry. 1994;57(8):886-96.

57. Pasinetti GM, Ungar LH, Lange DJ, Yemul S, Deng $H$, Yuan $X$, et al. Identification of potential CSF biomarkers in ALS. Neurology. 2006;66(8):1218-22.

58. Appel SH. A unifying hypothesis for the cause of amyotrophic lateral sclerosis, parkinsonism, and Alzheimer disease. Ann Neurol. 1981;10(6):499505.

59. Plaitakis A, Constantakakis E, Smith J. The neuroexcitotoxic amino acids glutamate and aspartate are altered in the spinal cord and brain in amyotrophic lateral sclerosis. Ann Neurol. 1988;24(3):446-9.

60. Chen H, Guo Y, Hu M, Duan W, Chang G, Li C. Differential expression and alternative splicing of genes in lumbar spinal cord of an amyotrophic lateral sclerosis mouse model. Brain Res. 2010;1340:52-69.

61. Chou SM, Norris FH. Amyotrophic lateral sclerosis: lower motor neuron disease spreading to upper motor neurons. Muscle Nerve. 1993;16(8):864-9.

62. Eisen AA. Comment on the lower motor neuron hypothesis. Muscle Nerve. 1993;16(8):870-1.

63. Jang SH, Seo JP. Aging of corticospinal tract fibers according to the cerebral origin in the human brain: a diffusion tensor imaging study. Neurosci Lett. 2015;585:77-81.

64. Mascalchi M, Salvi F, Valzania F, Marcacci G, Bartolozzi C, Tassinari CA. Corticospinal tract degeneration in motor neuron disease. AJNR Am J Neuroradiol. 1995;16(4 Suppl):878-80.

65. Yin H, Cheng SH, Zhang J, Ma L, Gao Y, Li D, et al. Corticospinal tract degeneration in amyotrophic lateral sclerosis: a diffusion tensor imaging and fibre tractography study. Ann Acad Med Singap. 2008;37(5):411-5. 